Research, Society and Development, v. 10, n. 15, e337101522756, 2021

(CC BY 4.0) | ISSN 2525-3409 | DOI: http://dx.doi.org/10.33448/rsd-v10i15.22756

\title{
Tratamento fisioterapêutico associado à utilização da toxina botulínica em pacientes com paralisia cerebral espástica: uma revisão integrativa
}

\author{
Physiotherapeutic treatment associated with the use of botulinum toxin in patients with spastic
} cerebral palsy: an integrative review

Tratamiento fisioterapéutico asociado al uso de toxina botulínica en pacientes con parálisis cerebral espástica: una revisión integradora

Recebido: 03/11/2021 | Revisado: 10/11/2021 | Aceito: 16/11/2021 | Publicado: 27/11/2021

\author{
Danielle Patricia de Souza \\ ORCID: https://orcid.org/0000-0001-8928-3497 \\ Universidade Tiradentes, Brasil \\ E-mail: danielles.souza84@gmail.com \\ Mariana Medeiros Gomes Peixoto \\ ORCID: https://orcid.org/0000-0002-4987-7239 \\ Universidade Tiradentes, Brasil \\ E-mail: mariana.mmgp@gmail.com \\ Sabrina de Melo Oliveira \\ ORCID: https://orcid.org/0000-0003-1024-4511 \\ Universidade Tiradentes, Brasil \\ E-mail: sabrina.brml@gmail.com \\ Breno Azevedo \\ ORCID: https://orcid.org/0000-0003-4917-8726 \\ Universidade Tiradentes, Brasil \\ E-mail: breno1azeved@gmail.com \\ Juliana Paulino Dantas da Silva Agranemam \\ ORCID: https://orcid.org/0000-0003-0114-2396 \\ Universidade Federal de Pernambuco, Brasil \\ E-mail: juliana.paulino@ufpe.br \\ Giovanna Cavalcanti \\ ORCID: https://orcid.org/0000-0003-3246-1306 \\ Universidade Federal de Pernambuco, Brasil \\ E-mail: giovannacavalcanti@gmail.com \\ Taylline Karolayne Gusmão de Oliveira \\ ORCID: https://orcid.org/0000-0002-0092-8701 \\ Universidade Federal de Pernambuco, Brasil \\ E-mail: Thayllineoliveirafisio@gmail.com \\ Bárbara Bernardo Figueirêdo \\ ORCID: https://orcid.org/0000-0002-4949-6268 \\ Universidade Federal de Pernambuco, Brasil \\ E-mail: prof.barbarabernardo@gmail.com
}

\begin{abstract}
Resumo
Introdução: A paralisia cerebral (PC) é caracterizada por uma lesão cerebral nos primeiros estágios do desenvolvimento, e pode estar associada a espasticidade. Tendo em vista a normalização do tônus muscular, a toxina botulínica (TB) é uma alternativa para proporcionar um relaxamento maior da musculatura em pacientes com espasticidade. $\mathrm{O}$ tratamento fisioterapêutico associado à utilização da toxina poderia potencializar o tratamento para a espasticidade. Objetivo: Revisar a literatura acerca do tratamento fisioterapêutico associado à utilização da TB em pacientes com paralisia cerebral espástica. Métodos: Foi realizada uma revisão de literatura nas bases de dados PubMed, LILACS, BVS e SciELO, de 2010 a 2020. Os descritores utilizados foram: paralisia cerebral, espasticidade, toxina botulínica e fisioterapia. Resultados: Foram encontraos 77 artigos, no entanto, foram excluidos 8 por estarem duplicados e 60 por não atenderem os critérios de inclusão, sendo incluidos 9 artigos nesta revisão. Nos estudos revisados, a TB quando associada a tratamentos fisioterapêuticos, promoveu o relaxamento da musculatura espastica, reduziu a dor, favoreceu o uso de órteses, aumentou a amplitude de movimento, melhorando a qualidade e expectativa de vida desses indivíduos. Considerações finais: O uso da TB associado a fisioterapia proporciona a melhora sobre a espasticidade e funcionalidade em pacientes com PC espástica.
\end{abstract}


Palavras-chave: Fisioterapia; Toxina botulínica; Toxinas botulínicas tipo A; Paralisia cerebral; Espasticidade muscular.

\begin{abstract}
Introduction: Cerebral palsy (CP) is characterized by brain damage in the early stages of development, and may be associated with spasticity. In view of the normalization of muscle tone, botulinum toxin (BT) is an alternative to provide greater relaxation of the muscles in patients with spasticity. Physiotherapy treatment associated with the use of toxin could enhance the treatment for spasticity. Objective: To review the literature on physical therapy treatment associated with the use of TB in patients with spastic cerebral palsy. Methods: A literature review was conducted in PubMed, LILACS, BVS and SciELO databases, from 2010 to 2020. The descriptors used were: cerebral palsy, spasticity, botulinum toxin and physical therapy. Results: 77 articles were found, however, 8 were excluded for being duplicates and 60 for not meeting the inclusion criteria, being included 9 articles in this review. In the reviewed studies, TB when associated with physical therapy treatments, promoted the relaxation of spastic muscles, reduced pain, favored the use of orthoses, increased the range of motion, improving the quality and life expectancy of these individuals. Final considerations: The use of TB associated with physical therapy provides improvement on spasticity and functionality in patients with spastic $\mathrm{CP}$.
\end{abstract}

Keywords: Physical therapy; Botulinum toxin; Botulinum toxins type A; Cerebral palsy; Muscle spasticity.

\title{
Resumen
}

Introducción: La parálisis cerebral (PC) se caracteriza por una lesión cerebral en las primeras etapas del desarrollo, y puede estar asociada a la espasticidad. Teniendo en cuenta la normalización del tono muscular, la toxina botulínica (TB) es una alternativa para proporcionar una mayor relajación de los músculos en pacientes con espasticidad. El tratamiento fisioterapéutico asociado al uso de la toxina podría mejorar el tratamiento de la espasticidad. Objetivo: Revisar la literatura sobre el tratamiento fisioterapéutico asociado al uso de TB en pacientes con parálisis cerebral espástica. Métodos: Se realizó una revisión de la literatura en las bases de datos PubMed, LILACS, BVS y SciELO, desde 2010 hasta 2020. Los descriptores utilizados fueron: parálisis cerebral, espasticidad, toxina botulínica y fisioterapia. Resultados: Se encontraron 77 artículos, sin embargo, 8 fueron excluidos por ser duplicados y 60 por no cumplir con los criterios de inclusión, siendo incluidos 9 artículos en esta revisión. En los estudios revisados, la TB cuando se asocia a tratamientos fisioterapéuticos, promueve la relajación de la musculatura espástica, reduce el dolor, favorece el uso de ortesis, aumenta la amplitud de movimiento, mejorando la calidad y la esperanza de vida de estos individuos. Consideraciones finales: El uso de TB asociado a la fisioterapia proporciona una mejora de la espasticidad y la funcionalidad en pacientes con PC espástica.

Palabras clave: Fisioterapia; Toxina botulínica; Toxinas botulínicas tipo A; Parálisis cerebral; Espasticidad muscular.

\section{Introdução}

A Paralisia Cerebral (PC) ou encefalopatia crônica não progressiva da infância, é consequência de uma lesão estática, que se constitui em um grupo de desordens permanentes no desenvolvimento do movimento durante o período pré, peri ou pós-natal que afeta o sistema nervoso central na fase de maturação estrutural e funcional, pode ser acompanhada por distúrbios sensoriais, cognitivos, comportamental, de comunicação e por problemas musculoesqueléticos (Rodríguez-Reyes et al., 2010; Figueiredo et al. 2021).

A PC pode ser classificada como tetraplegia, hemiplegia e diplegia, de acordo com a localização corporal do comprometimento neuromortor, e com as características clínicas dominantes em atáxica, hipotônica, atetósica, espástica e mista, sendo a forma espástica a mais comum (Chaléat-Valayer et al., 2011).

A espasticidade é definida como aumento da tensão do músculo, com os reflexos profundos intensificados decorrente de um exagero do reflexo de estiramento muscular (Sáinz-Pelayo, Murillo, Benito-Penalva, 2020). O aumento do tônus pode afetar de maneira contrária ao desenvolvimento motor, podendo levar a padrões de movimentos anormais, deformidades musculoesqueléticas e atrasos na aquisição das habilidades motoras e é considerada um dos maiores obstáculos para a reabilitação neurológica (Chaléat-Valayer et al. 2011; Gomes \& Goli, 2013).

Dentre várias terapias utilizadas para tratamento da espasticidade, a Toxina Botulínica do tipo A (TB A) vem sendo utilizada, possui efeitos colaterais mais reduzidos e efeito evidentes na diminuição da espasticidade. A TB é uma proteína 
produzida pela bactéria anaeróbia Clostridium Botulinum. São conhecidos 8 tipos de neurotoxinas das quais sete são neurotóxicas (de A a G). Destas o tipo A é considerada a mais potente e por este motivo é a mais usada clinicamente (Cesa et al. 2020; Sáinz-Pelayo et al., 2020).

Entre os tratamentos da PC espástica, a fisioterapia tem por objetivo inibir a atividade reflexa patológica, normalizar o tônus muscular, ajudando na postura e volta aos padrões normais, visando promover a funcionalidade, com técnicas para diminuir a hipertonia muscular, minimizar os problemas secundários, como encurtamentos e contraturas musculares (Gallagher et al. 2015).

Há recomendação para que a TB A seja utilizada em conjunto com a fisioterapia, visto que a aplicação da TB A isoladamente pode não garantir total eficácia na redução da espasticidade de um indivíduo com a PC (Gomes \& Goli, 2013). A sua efetividade pode ser diminuída ou aumentada, isso dependerá se os locais de aplicação forem ou não estimulados e trabalhados (Carbonari de Faria et al. 2001; Resende et al. 2005).

A melhora do uso da TB A pode relacionar-se com o uso de um programa de reabilitação intensivo e uso de tratamento dinâmico depois da injeção. Em questão ao tempo, para conseguir um efeito mais prolongado e permanente, é absolutamente necessário que o paciente receba fisioterapia que potencialize a mobilidade e a força da musculatura agonista e antagonista e alcance os efeitos da desnervação em conjunto com o resto dos grupos musculares (Aguilar-Rebolledo et al. 2001).

Portanto o objetivo deste estudo foi realizar uma revisão integrativa para unir informações sobre as evidências do tratamento fisioterapêutico associado à utilização da TB A em pacientes com Paralisia Cerebral Espástica.

\section{Metodologia}

Trata-se de uma revisão integrativa, com buscas nas bases de dados eletrônicas: Nacional Library of Medicine (PubMed), Literatura Latino-Americana e do Caribe em Ciências da Saúde (LILACS), Biblioteca Virtual de Saúde (BVS) e Scientific Electronic Library Online (SciELO).

Esse estudo foi construído respeitando as seguintes etapas: a) elaboração da pergunta norteadora; b) busca na literatura; c) coleta de dados; d) análise crítica dos estudos incluídos; e) discussão dos resultados; f) apresentação da revisão. Os critérios do sistema descritor utilizado nesta revisão foram realizados seguindo os Medical Subject Headings (MeSH) e Health Sciences Descriptors (DeCS) e os operadores booleanos, como "AND" e "OR" foram usados, por meio do sistema de busca, utilizando como descritores as palavras-chave "paralisia cerebral" e "espasticidade" combinadas com "toxina botulínica" e "fisioterapia".

A pesquisa também foi realizada por meio de consulta dos mesmos descritores em inglês "cerebral palsy" e "spasticity" combinadas com "botulinum toxin" e "physiotherapy". A seleção buscou artigos durante um período de 10 anos, entre 2010 e 2020, nas línguas portuguesa, espanhola e inglesa. A busca aconteceu no período de 09 a 26 de março de 2020.

Todos os processos de busca, seleção e avaliação dos artigos foram realizados de forma a analisar completamente e detalhadamente todos os estudos que atendiam aos critérios de inclusão. Os resultados obtidos foram avaliados por dois avaliadores, que verificaram a concordância entre os pares para inclusão dos artigos. Foram selecionados estudos que atendiam aos critérios de inclusão: tratavam sobre a associação do tratamento fisioterapêutico, ou aplicação de técnicas de fisioterapia com a aplicação da toxina botulínica A em indivíduos com paralisia cerebral para o controle da espasticidade. 
Research, Society and Development, v. 10, n. 15, e337101522756, 2021

(CC BY 4.0) | ISSN 2525-3409 | DOI: http://dx.doi.org/10.33448/rsd-v10i15.22756

Foram incluídos estudos transversais, ensaios clínicos, relatos de caso e foram excluídos os que não atendiam os critérios citados e as revisões de literatura acerca do tema. Quando a leitura dos resumos não se mostrou satisfatória para o entendimento do contexto, foi acessado o artigo completo para uma boa compreensão e interpretação.

Quando houve questionamentos sobre a inclusão do artigo, ele foi lido de forma independente pelos pesquisadores e o consenso entre eles foi adotado. Foram lidos antecipadamente os resumos dos artigos identificados nas bases de dados citadas acima, de forma a identificar os métodos propostos, aplicados e discutidos por cada autor.

\section{Resultados}

O fluxograma com os resultados da busca encontra-se descrito na Figura 1 e nos Quadros 1 e 2 exibe-se as características dos estudos selecionados, explicitando o tipo de estudo, amostra, faixa etária dos participantes, método e resultados encontrados.

A TB A é a mais utilizada para o tratamento da espasticidade em crianças com PC (Schasfoort et al. 2018). A TB quando associada à fisioterapia para controle da espasticidade se mostra bastante eficaz e os efeitos do uso da mesma no tratamento da espasticidade são evidenciados na escala de Ashworth e presumem potencializar o tratamento fisioterapêutico (Carbonari de Faria et al. 2001).

O uso da toxina botulínica associado com a fisioterapia pode evitar ou retardar possíveis cirurgias que seriam realizadas precocemente, gerando um benefício em valores que seriam gastos com internamento, tratamento cirúrgico e pósoperatório. Tendo em vista as características dos pacientes com PC, como alterações de controle motor e sensibilidade, necessitando de auxílio por maior parte do tempo, o que gera e custos elevados, impactando na saúde pública e no sistema previdenciário (Schasfoort et al. 2018).

A necessidade de acelerar o entendimento do mecanismo de ação da toxina botulínica em pacientes com espasticidade provocada por paralisia cerebral é importante para que sejam traçadas condutas fisioterapêuticas focadas para o tratamento de crianças com paralisia cerebral do tipo espástica (Resende et al. 2005). 
Research, Society and Development, v. 10, n. 15, e337101522756, 2021

(CC BY 4.0) | ISSN 2525-3409 | DOI: http://dx.doi.org/10.33448/rsd-v10i15.22756

Figura 1 - Fluxograma das fases de identificação, triagem e seleção de artigos sobre Tratamento fisioterapêutico associado à utilização da toxina botulínica em pacientes com paralisia cerebral espástica.

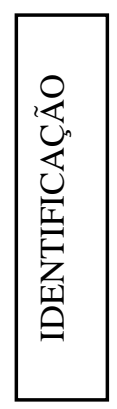

Artigos científicos identificados nas buscas em bases de dados virtuais: PubMed,

SciELO, BVS e LILACS.

$(\mathrm{n}=77)$

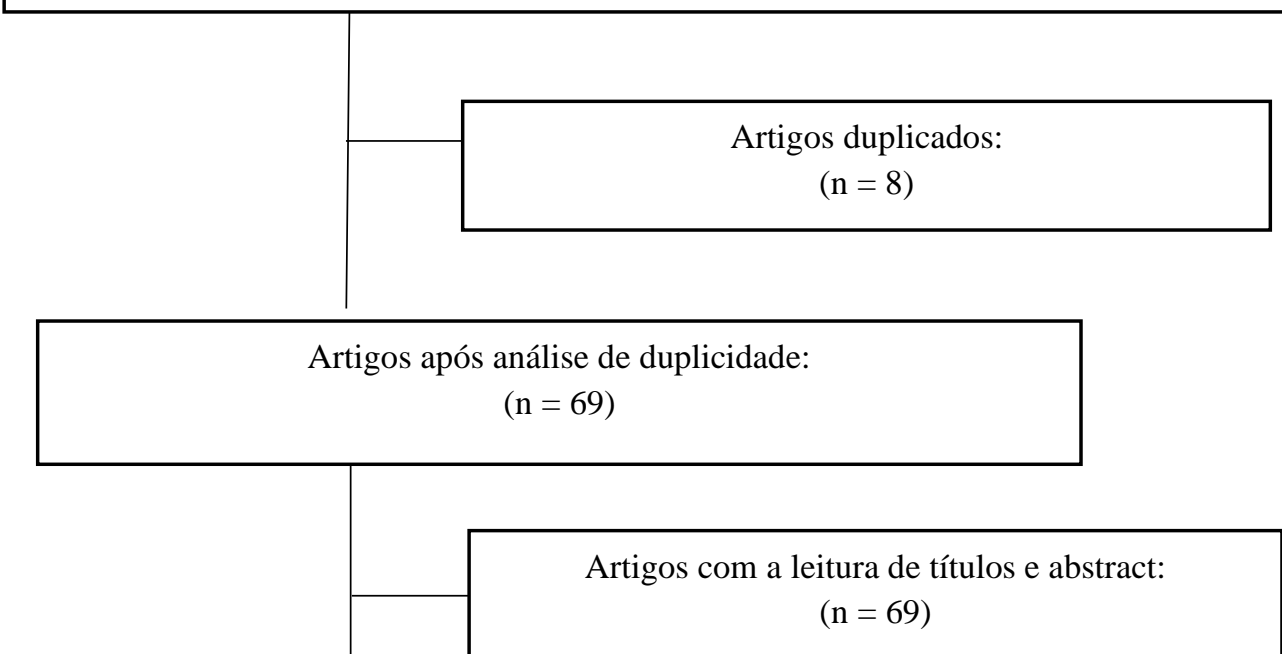

Artigos lidos na íntegra pelos avaliadores para elegibilidade:

$(n=69)$

Número de artigos excluídos: $(\mathrm{n}=59)$

Desfecho principal não analisado: $(n=13)$

Intervenção incompatível: $(n=16)$

Amostra incompatível: $(\mathrm{n}=15)$

Comparação incompatível: $(n=6)$

Tipo de estudo incompatível: $(\mathrm{n}=9)$

Fonte: Dados da pesquisa. 
Research, Society and Development, v. 10, n. 15, e337101522756, 2021

(CC BY 4.0) | ISSN 2525-3409 | DOI: http://dx.doi.org/10.33448/rsd-v10i15.22756

Quadro 1 - Características dos artigos sobre o tipo do estudo, amostra e faixa etária dos participantes.

\begin{tabular}{|c|c|c|c|}
\hline Autor/Ano & Tipo do estudo & Amostra & Faixa etária \\
\hline $\begin{array}{l}\text { Aguilar-Reboledo et } \\
\text { al., } 2001\end{array}$ & Estudo aberto prospectivo. & 27 pacientes com PC espástica. & 3 a 23 anos \\
\hline $\begin{array}{l}\text { Chaleat-Valayer et } \\
\text { al., } 2011\end{array}$ & Estudo Observacional prospectivo. & 282 crianças com PC espástica & 3 a 6 anos \\
\hline $\begin{array}{l}\text { Esquenazi et al., } \\
\qquad 2020\end{array}$ & $\begin{array}{l}\text { Estudo duplo cego, de único ciclo, } \\
\text { randomizado e controlado por placebo }\end{array}$ & 388 pacientes com PC espástica & 18 a 80 anos \\
\hline Faria et al.,2001 & estudo prospectivo (causa e efeito) & $\begin{array}{c}14 \text { crianças com PC hemiparético } \\
\text { espástica. }\end{array}$ & 3 a 9 anos \\
\hline Franco et al., 2006 & Estudo observacional transversal & 10 crianças com PC espástica. & 2 a 7 anos \\
\hline Gomes et al., 2013 & Estudo transversal & 3 pacientes com PC espástica. & 3 a 7 anos \\
\hline Resende et al., 2005. & Caso clínico & 1 criança com PC espástica. & 5 anos \\
\hline $\begin{array}{l}\text { Rodriguez - Reyes } \\
\quad \text { et al., } 2010\end{array}$ & $\begin{array}{l}\text { Ensaio clínico randomizado } \\
\text { multicêntrico }\end{array}$ & $\begin{array}{c}10 \text { crianças com PC espasticidade de } \\
\text { membros superiores. }\end{array}$ & 3 a 6 anos \\
\hline $\begin{array}{l}\text { Schasfoort et al., } \\
2018\end{array}$ & $\begin{array}{l}\text { Estudo pragmaticamente projetado, } \\
\text { simples-cego e multicêntrico }\end{array}$ & 65 crianças com PC espástica & 4 a 12 anos \\
\hline
\end{tabular}

Fonte: Dados da pesquisa. 
Quadro 2 - Características dos artigos sobre o tratamento fisioterapêutico associado à utilização da toxina botulínica em pacientes com paralisia cerebral espástica.

\begin{tabular}{|c|c|c|}
\hline Autor/Ano & Métodos & Resultados \\
\hline $\begin{array}{l}\text { Aguilar- } \\
\text { Reboledo } \text { et } \\
\text { al., } 2001\end{array}$ & $\begin{array}{l}\text { A TB A foi aplicada nos músculos adutores de coxa, isquiotibiais, } \\
\text { gêmeos, sóleo e tibial posterior. Os efeitos foram avaliados por meio } \\
\text { da Escala de Ashworth modificada. Período: } 3 \text { anos. }\end{array}$ & $\begin{array}{l}\text { Foi possível detectar diferenças palpáveis no tônus } \\
\text { muscular, na funcionalidade do membro, na marcha } \\
\text { e no posicionamento dos pés através da TB A } \\
\text { associada ao tratamento fisioterapêutico. }\end{array}$ \\
\hline $\begin{array}{l}\text { Chaleat- } \\
\text { Valayer et } \\
\text { al., } 2011\end{array}$ & $\begin{array}{l}\text { A TB A foi aplicada nos membros inferiores. Os efeitos foram } \\
\text { avaliados por meio da Escala Visual Analógica (EVA), Escala de } \\
\text { Ashworth modificada, Questionário de Avaliação Funcional de } \\
\text { Gillette e Medida de Função Motora Grossa-66. Período: Foram } \\
\text { avaliadas mensalmente por } 12 \text { meses. }\end{array}$ & $\begin{array}{l}\text { O tratamento com injeções de TB A administrada em } \\
\text { diferentes localizações musculares com doses } \\
\text { maiores que habitualmente recomendadas associadas } \\
\text { ao tratamento fisioterapêutico, gessos e órteses } \\
\text { reduziu a espasticidade e melhorou a amplitude de } \\
\text { movimento articular, o padrão de marcha e a } \\
\text { capacidade de realização de movimentos. }\end{array}$ \\
\hline $\begin{array}{l}\text { Esquenazi et } \\
\text { al., } 2020\end{array}$ & $\begin{array}{l}\text { Os pacientes foram divididos em grupo de aplicação da TB A e grupo } \\
\text { placebo. As aplicações foram feitas nos músculos sóleo, } \\
\text { gastrocnêmios e foram associadas a sessões de fisioterapia durante } 1 \\
\text { mês. A avaliação da espasticidade foi realizada através da Escala de } \\
\text { Tardieu. Período: De } 12 \text { a } 24 \text { semanas }\end{array}$ & $\begin{array}{l}\text { As análises demonstram que um único ciclo de } \\
\text { tratamento com a TB A associada a tratamento } \\
\text { fisioterapêutico mostra melhoras nos parâmetros de } \\
\text { tônus muscular e espasticidade. }\end{array}$ \\
\hline $\begin{array}{l}\text { Faria et } \\
\text { al.,2001 }\end{array}$ & $\begin{array}{l}\text { TB A foi aplicada nos músculos solear e gastrocnêmios. E a } \\
\text { cinesioterapia com adequação do tônus muscular, alongamentos do } \\
\text { tríceps sural, facilitação e fortalecimento do tibial anterior, } \\
\text { transferência de peso no membro lesado, velocidade e cadência da } \\
\text { marcha e orientações. Período: } 1 \text { a } 3 \text { meses. }\end{array}$ & $\begin{array}{l}\text { A TB A contribuiu como agente facilitador para o } \\
\text { tratamento cinesioterápico, observando-se melhora } \\
\text { do equino dinâmico do tornozelo num curto período } \\
\text { de tempo. O tratamento cinesioterápico e o uso da } \\
\text { TB A em músculos sóleos e gastrocnêmios } \\
\text { associados a técnicas de alongamentos, mobilizações } \\
\text { e fortalecimento são úteis na facilitação da } \\
\text { normalização da marcha. }\end{array}$ \\
\hline $\begin{array}{c}\text { Franco et al., } \\
2006\end{array}$ & $\begin{array}{l}\text { Doses injetáveis da TB A em gastrocnêmios, direito e esquerdo, } \\
\text { seguindo de sessões de fisioterapia. Foram coletados dados da } \\
\text { atividade reflexa, trofismo muscular, graduação do tônus muscular } \\
\text { dos gastrocnêmios na Escala de Ashworth modificada, grau de ADM } \\
\text { do tornozelo com goniometria, avaliação da marcha e do engatinhar } \\
\text { de acordo com a escala validade de atividades funcionais dinâmicas. }\end{array}$ & $\begin{array}{l}\text { A TB A combinada a fisioterapia mostrou ser útil no } \\
\text { tratamento da espasticidade de paciente portadores } \\
\text { de PC espástica, quando aplicada nos gastrocnêmios, } \\
\text { aumentando a amplitude de movimento do tornozelo, } \\
\text { reduzindo a hipertonia muscular. As duas } \\
\text { intervenções associadas facilitam as atividades } \\
\text { funcionais através da graduação na escala de } \\
\text { atividades funcionais dinâmicas, contribuindo para a } \\
\text { melhora dos pacientes. }\end{array}$ \\
\hline $\begin{array}{c}\text { Gomes et al., } \\
2013\end{array}$ & $\begin{array}{l}\text { Foi feita aplicação da TB A associada a fisioterapia com padrão de } \\
\text { inibição, manobras para diminuir a hipertonia, facilitação do controle } \\
\text { cervical, cintura escapular e do ro rolar. } \\
\text { Foi utilizada a Escala de Ashworth modificada para avaliação do } \\
\text { tônus muscular. }\end{array}$ & $\begin{array}{l}\text { A aplicação da TB A associada a fisioterapia foi } \\
\text { eficaz na facilitação do controle cervical e da cintura } \\
\text { escapular. }\end{array}$ \\
\hline $\begin{array}{l}\text { Resende et } \\
\text { al., } 2005 .\end{array}$ & $\begin{array}{l}\text { Uso da TB A no tibial posterior e tríceps sural associado a } \\
\text { cinesioterapia, } 2 \text { vezes por semana e hidroterapia, } 3 \text { vezes na semana. } \\
\text { A Escala de Ashworth modificada foi utilizada para avaliar a } \\
\text { espasticidade. Período: Primeira avaliação antes da TB A e a segunda } \\
\text { após } 15 \text { dias da aplicação em que se foi realizado o tratamento } \\
\text { fisioterapêutico associado ao uso de órtese. }\end{array}$ & $\begin{array}{l}\text { A TB A associada a hidroterapia e cinesioterapia } \\
\text { foram eficazes, houve melhora da marcha, } \\
\text { mobilidade articular do membro inferior direito e } \\
\text { melhora da espasticidade. }\end{array}$ \\
\hline
\end{tabular}




\begin{tabular}{|c|c|c|}
\hline $\begin{array}{c}\text { Rodriguez - } \\
\text { Reyes et al., } \\
2010\end{array}$ & $\begin{array}{l}\text { A TB A foi injetada em músculos espásticos dos membros superiores. } \\
\text { Foram realizadas } 10 \text { sessões de fisioterapia e terapia ocupacional, } \\
\text { seguidas por } 10 \text { sessões de estimulação elétrica neuromuscular nos } \\
\text { extensores do punho (músculos antagonistas) associada a fisioterapia } \\
\text { com neurofacilitação (método Bobath), mobilização assistida e } \\
\text { alongamentos de membros superiores. A função manual foi avaliada } \\
\text { pelo Teste de função manual de Jebsen Taylor, o grau de } \\
\text { espasticidade foi avaliado pela escada modificada de Ashworth, a } \\
\text { ADM do punho foi medida por equipamento de análise de } \\
\text { movimento. Período: } 10 \text { sessões }\end{array}$ & $\begin{array}{l}\text { Evidenciou o efeito positivo do tratamento } \\
\text { fisioterapêutico associado a aplicação da toxina } \\
\text { botulínica na função manual, na ADM do punho e na } \\
\text { melhora da espasticidade. }\end{array}$ \\
\hline $\begin{array}{l}\text { Schasfoort et } \\
\text { al., } 2018\end{array}$ & $\begin{array}{l}41 \text { crianças receberam toxina botulínica tipo A mais fisioterapia } \\
\text { intensiva e } 24 \text { receberam apenas tratamento fisioterapêutico intensivo. } \\
\text { Avaliou-se a função motora grossa com a escala GMFM- } 66 \text {. Grupo I } \\
\text { = Fisioterapia mais TB A e Grupo } 2=\text { TB A. Período: } 12 \text { semanas }\end{array}$ & $\begin{array}{l}\text { As injeções de TB A não melhoraram os efeitos } \\
\text { clínicos sozinhas, precisando de intervenção } \\
\text { fisioterapêutica para melhora do quadro de } \\
\text { espasticidade. }\end{array}$ \\
\hline
\end{tabular}

Legenda: ADM: Amplitude de Movimento; ADV: Atividades de Vida Diária; EVA: Escala Visual Analógica; PC: Paralisia Cerebral; TBA: Toxina Botulínica tipo A. Fonte: Dados da pesquisa.

\section{Discussão}

A TB A tem sido o método de escolha para reduzir a espasticidade em pacientes com paralisia cerebral espástica ou com componente espástico. A redução da espasticidade pode contribuir para uma melhora na função dos membros superiores e inferiores, nessa população (Esquezani et al. 2020).

O progresso da função em pacientes pós aplicação da TB A está diretamente associada a intervenções fisioterapêuticas, a reabilitação faz com que os benefícios da redução da espasticidade após aplicação da toxina possam ser potencializados através de exercícios de fortalecimento, alongamento e eletroestimulação, tendo em vista a melhoria do desempenho funcional (Rodríguez-Reyes et al., 2010). A droga é injetada pela equipe médica, e a potencialização dos ganhos se dá pela equipe de fisioterapia, que prosseguirá com o tratamento após as aplicações (Resende et al. 2005).

A utilização da toxina tem um número reduzido de efeitos colaterais, o que facilita a indicação ${ }^{6}$. Embora seja um procedimento terapêutico favorável na redução da espasticidade, alguns fatores limitam sua eficácia, um desses fatores é em relação ao tempo de duração dos efeitos da TB A, alguns estudos mostram que o tempo de efeito dessa terapêutica surgem entre 24 e 72 horas após e tem duração de 3 a 6 meses. Após esse período, uma redução dos efeitos é encontrada, com consequente retorno gradual da espasticidade sendo essencial outras aplicações para que o efeito possa se prolongar (Carbonari de Faria et al. 2001).

A TB A associada a cinesioterapia tem como objetivo melhorar a funcionalidade e as habilidades do paciente, inibir a atividade reflexa anormal, proporcionar a redução do tônus muscular, aprimorar os padrões de movimento normal, melhorar a força, a flexibilidade, a amplitude de movimento, de forma a facilitar o desenvolvimento motor. Além disso, essa associação dos exercícios fisioterapêuticos com as aplicações toxina botulínica tem demonstrado efetividade e segurança (Gomes \& Goli, 2013).

Segundo Resende et al. (2005). o aumento no tônus dos músculos normais induz a redução do comprimento muscular anatômico, assim a redução do tônus dos músculos espásticos como o uso da TB A ocasionou o aumento do estiramento longitudinal passivo dos músculos ocorrendo a restauração do comprimento longitudinal normal.

A toxina botulínica do tipo A é utilizada para redução de contraturas musculares severas em pacientes com PC do tipo espástica e a cinesioterapia tem efeito positivo sobre a amplitude movimento, apresentando benefícios para prevenção de contraturas musculares, sendo assim, são opções terapêuticas eficientes para a manutenção da amplitude de movimento, tanto 
em membros superiores quanto nos membros inferiores (Rodríguez-Reyes et al., 2010; Sáinz-Pelayo, Murillo, Benito-Penalva, 2020).

Os estudos revisados, apresentaram procedimentos metodológicos e tamanho da amostra distintos, a maioria dos estudos consideraram os efeitos da aplicação da TB A em um período de 3 meses até 6 meses, e é neste tempo que os efeitos são potencializados. Após este período, os efeitos começam a diminuir sendo necessária uma nova aplicação para que o tratamento fisioterapêutico alcance o objetivo esperado.

No estudo de Aguillar-Reboledo et al. (2001), 27 pacientes foram avaliados, onde todos eles haviam recebido reabilitação prévia. A indicação de tratamento foi para reduzir a espasticidade e distonia, melhorar a funcionalidade, evitar ou adiar a cirurgia corretiva e melhorar a higiene e / ou o vestuário. A infiltração do TB A foi realizada com ou sem controle eletromiográfico, em um ou dois pontos do ventre de cada músculo. Os resultados foram avaliados pela escala de Ashworth onde foram validados quanto a eficiência da TB A associada a tratamento fisioterapêutico.

No estudo de Sáinz-Pelayo (2020), das 282 crianças avaliadas, 215 crianças usavam órteses (76\%) e 57 (20\%) já haviam feito gesso seriado. Apenas 44 crianças (16\%) haviam recebido medicamentos antiespásticos anteriormente, e 122 (43\%) já haviam sido tratadas com TB A. O tratamento com a TB A foi administrado em diferentes localizações musculares de uma única vez e em doses maiores do que as recomendadas pelas autoridades de saúde francesas, essas crianças eram tratadas paralelamente por fisioterapia, gessos e órteses (Sáinz-Pelayo et al., 2020).

As injeções reduziram a espasticidade e melhoraram a amplitude de movimento articular, o padrão de marcha e a capacidade de movimento. O estudo concluiu que as injeções de TB A contribuem no tratamento da espasticidade em crianças com PC como tratamento conservador, seguro e eficiente, possibilitando melhora funcional e alívio da dor (Sáinz-Pelayo et al. 2020).

Esquenazi et al. (2020), em seu estudo, teve como objetivo avaliar a segurança e eficácia da aplicação da TB A em pacientes com PC espástica quando associada a fisioterapia. Os pacientes foram divididos em aplicação da TB A e em placebo. $\mathrm{O}$ estudo mostra que os pacientes tratados com a TB A mostraram melhora no tônus muscular e na espasticidade quando associada a fisioterapia, se tornando assim segura e eficaz.

O trabalho de Faria et al. (2001), teve como objetivo avaliar a atuação da cinesioterapia associada ao efeito da TB A por meio da análise da deambulação em 14 crianças com PC do tipo hemiparética. Os pacientes foram divididos aleatoriamente em dois grupos: grupo I (TBA) - recebeu aplicação da toxina nos músculos sóleos e gastrocnêmios e também intervenção fisioterapêutica após as injeções; já o grupo II (controle) recebeu somente a cinesioterapia, sendo esta igual à do grupo I.

Todos os pacientes do grupo I (100\%) apresentaram melhora da posição do tornozelo nas diferentes fases da marcha, do comprimento do passo e da velocidade de deambulação, enquanto apenas 28,6\% obtiveram melhora no grupo II. Ainda assim, no grupo II não ocorreu melhora de todas as medidas avaliadas e o ganho em algumas destas aconteceu em um maior período de tempo (Carbonari de Faria et al. 2001).

Franco et al. (2006), em sua pesquisa, tiveram como objetivo avaliar o grau de amplitude de movimento da articulação do tornozelo, em crianças com PC, após a aplicação de TB A nos músculos gastrocnêmios seguida de fisioterapia. Foram estudadas 10 crianças, com faixa etária de 2 a 7 anos, com presença de equinismo bilateral, que é um distúrbio da marcha bastante comum em crianças com paralisia cerebral.

O estudo concluiu que a toxina botulínica tipo A combinada ao tratamento fisioterapêutico é um método eficaz no processo de reabilitação de crianças que apresentam PC espástica (Franco et al., 2006).

O estudo de Gomes \& Golin (2013), teve por objetivo descrever o tratamento fisioterapêutico associado a TB A em crianças com PC, 3 pacientes do sexo feminino com faixa etária de 3 a 8 anos, com diagnostico de PC tipo tetraparesia 
espástica participaram do estudo. Concluiu-se que quanto mais precoce a intervenção da fisioterapia associada a aplicação da TB A, mais eficaz as respostas clínicas.

Resende et al. (2005), realizou um estudo com o objetivo de avaliar a eficácia da aplicação da toxina botulínica associada a fisioterapia em uma criança com paralisia cerebral tipo espástica de 5 anos de idade. Ela foi submetida a duas avaliações, a primeira antes da aplicação da TB A no músculo tibial posterior e tríceps sural e a segunda após 15 dias associada a tratamento fisioterapêutico e uso de órtese. A fisioterapia foi realizada em solo e hidroterapia. Os dados foram analisados através do test t de Student.

Rodríguez-Reyes et al., (2010). em sua pesquisa, tiveram como objetivo avaliar os efeitos da aplicação da TB A associada a fisioterapia e informar cálculos de tamanho de amostra para um ensaio clínico randomizado. Foram estudadas 10 crianças portadoras de PC espástica em membro superior. A TB A foi injetada em músculos espásticos dos membros superiores de 10 crianças.

Eles receberam 10 sessões de fisioterapia e terapia ocupacional, seguidas por 10 sessões de estimulação elétrica neuromuscular nos extensores do punho (músculos antagonistas) associada a fisioterapia com neurofacilitação (método Bobath), mobilização assistida e alongamento de membros superiores e grupo dos músculos torácicos, com atividades manuais e exercícios, reforço de propriocepção corporal e AVDs (Rodríguez-Reyes et al., 2010). O estudo concluiu que a toxina botulínica tipo A combinada ao tratamento fisioterapêutico de estimulação elétrica neuromuscular (EENM) é um método eficaz no processo de reabilitação de crianças que apresentam PC (Rodríguez-Reyes et al., 2010).

Schasfoort et al. (2018), teve por objetivo analisar o uso da TB A em crianças com PC espástica e avaliar os efeitos das injeções antes do período do período de tratamento fisioterapêutico intensivo. Foram estudadas 65 crianças com faixa etária de 4 a 12 anos, com diagnóstico de PC espástica, com comprometimento nos membros inferiores (unilateral ou bilateral), classificado nos níveis I - III do Sistema de Classificação da Função Gross Motor. O estudo concluiu que as injeções de TB A não melhoraram os efeitos clínicos sozinhas, sendo assim é necessária intervenção fisioterapêutica para melhora do quadro de espasticidade (Faria \& Silva, 2014).

O uso da TB A na paralisia cerebral justifica-se pôr o uso da mesma gerar uma diminuição da espasticidade, a partir daí abre-se a possibilidade terapêutica para intervenções, já que a toxina botulínica tem como principal contribuição o controle temporário da hipertonia muscular, melhorando a capacidade motora e as habilidades funcionais e evitando a formação de contraturas.

Esse controle acontece devido ao fato de que a TB A age sobre os receptores de acetilcolina na junção neuromuscular, inibindo a liberação de acetilcolina na membrana pré-sináptica, o que provoca a paralisia do músculo através do bloqueio da via de neurotransmissão. Programas de tratamento cinesioterapêuticos são apontados pela equipe de saúde como fundamentais após a injeção da droga, eles têm como objetivo adequar o tônus muscular, alongar e fortalecer o músculo (Multani et al. 2019; Serrera-Figallo et al. 2020).

\section{Considerações Finais}

Nos estudos revisados, a fisioterapia associada a toxina botulínica A proporcionou um melhor controle da espasticidade, potencializando os ganhos, como a melhora da amplitude de movimento e da funcionalidade em pacientes com paralisia cerebral espástica ou com componente espástico. Contudo, novas pesquisas, como a realização de ensaios clínicos com critérios bem definidos devem ser realizados para melhores confirmações dos resultados encontrados nos estudos aqui revisados. 
Research, Society and Development, v. 10, n. 15, e337101522756, 2021

(CC BY 4.0) | ISSN 2525-3409 | DOI: http://dx.doi.org/10.33448/rsd-v10i15.22756

\section{Referências}

Aguilar-Rebolledo, F., Hernández-Sánchez, J., Rayo-Mares, D., Soriano, F. F., García-Muñoz, L., Ruiz-Ponce, J., Garrido-Ramírez, E., (2001) Toxina botulínica como tratamiento de la espasticidad y distonía en la parálisis cerebral infantil. Gac Med Mex. ;137(5):403-411.

Carbonari de Faria, T. C., Masiero. D., Spósito, M., Saad, M. (2001). A avaliação do uso da toxina botulínica A e da cinesioterapia na melhora da marcha do portador de paralisia cerebral do tipo hemiparético. ActraFisiatrica.8(3), 1-6.

Cesa, CC, Motta, HB, \& Brandão, L. (2020). Análise conversacional de crianças com paralisia cerebral: estudo de casos múltiplos. Research, Society and Development , 9 (6), e133963580. https://doi.org/10.33448/rsd-v9i6.3580

Chaléat-Valayer, E., Parratte, B., Colin, C., Denis, A., Oudin, S., Bérard, C., Bernard, J. C., Bourg, V., Deleplanque, B., Dulieu, I., Evrard, P., Filipetti, P., Flurin, V., Gallien, P., Héron-Long, B., Hodgkinson, I., Husson, I., Jaisson-Hot, I., Maupas, E., Meurin, F., ... Marque, P. (2011). A French observational study of botulinum toxin use in the management of children with cerebral palsy: BOTULOSCOPE. European journal of paediatric neurology : EJPN : official journal of the European Paediatric Neurology Society, 15(5), 439-448. https://doi.org/10.1016/j.ejpn.2010.04.006

Esquenazi, A., Stoquart, G., Hedera, P., Jacinto, L. J., Dimanico, U., Constant-Boyer, F., Brashear, A., Grandoulier, A. S., Vilain, C., Picaut, P., \& Gracies, J. M. (2020). Efficacy and Safety of AbobotulinumtoxinA for the Treatment of Hemiparesis in Adults with Lower Limb Spasticity Previously Treated With Other Botulinum Toxins: A Secondary Analysis of a Randomized Controlled Trial. PM \& $R$ : the journal of injury, function, and rehabilitation, 12(9), 853860. https://doi.org/10.1002/pmrj. 12348

Faria, S. S., \& Pedro Leme Silva, P. L. S. P. L. S. (2014). Revisão Sistemática sobre Tratamento Medicamentoso para Dor no Membro Fantasma. Revista Neurociências, 22(2), 177-188. https://doi.org/10.34024/rnc.2014.v22.8091

Figueiredo, B. Q. de, Ricardo Neto, A.., Nogueira, C. F. R., Fernandes, R. A., \& Almeida, M. G. (2021). Tratamento das sequelas do Acidente Cerebrovascular (AVC) com toxina botulínica: uma revisão da literatura. Research, Society and Development , 10 (8), e56610817880. https://doi.org/10.33448/rsd-v10i8.17880

Franco, C. Buarque, P., Larissa, de C., Pontes, L. da S., \& Sousa, E. de J. (2006). Avaliação da amplitude articular do tornozelo em crianças com paralisia cerebral após a aplicação de toxina botulínica seguida de fisioterapia. Revista Paraense de Medicina, 20(3), 43-49. Recuperado em 01 de novembro de 2021, de http://scielo.iec.gov.br/scielo.php?script=sci_arttext\&pid=S0101-59072006000300009\&lng=pt\&tlng=pt.

Gallagher, S., Phadke, C. P., Ismail, F., \& Boulias, C. (2015). Physical therapy for an adult with chronic stroke after botulinum toxin injection for spasticity: a case report. Physiotherapy Canada. Physiotherapie Canada, 67(1), 65-68. https://doi.org/10.3138/ptc.2013-73

Gomes, C. de O., \& Golin, M. O. (2013). Tratamento Fisioterapêutico Na Paralisia Cerebral Tetraparesia Espástica, Segundo Conceito Bobath. Revista Neurociências, 21(2), 278-285. https://doi.org/10.34024/rnc.2013.v21.8293

Multani, I., Manji, J., Hastings-Ison, T., Khot, A., \& Graham, K. (2019). Botulinum Toxin in the Management of Children with Cerebral Palsy. Paediatric drugs, 21(4), 261-281. https://doi.org/10.1007/s40272-019-00344-8.

Resende, C. M. G., do, Nascimento. V. F., \& S., Leite. J. M. R. (2005). Eficácia da toxina botulínica tipo-A associada a fisioterapia em uma criança hemiplégica espástica. Revista Neurociências, 13(1), 17-20. https://doi.org/10.34024/rnc.2005.v13.8840

Rodríguez-Reyes, G., Alessi-Montero, A., Díaz-Martínez, L., Miranda-Duarte, A., \& Pérez-Sanpablo, A. I. (2010). Botulinum toxin, physical and occupational therapy, and neuromuscular electrical stimulation to treat spastic upper limb of children with cerebral palsy: a pilot study. Artificial organs, 34(3), 230-234. https://doi.org/10.1111/j.1525-1594.2009.00768.x

Sáinz-Pelayo, M. P., Albu, S., Murillo, N., \& Benito-Penalva, J. (2020). Espasticidad en la patología neurológica. Actualización sobre mecanismos fisiopatológicos, avances en el diagnóstico y tratamiento [Spasticity in neurological pathologies. An update on the pathophysiological mechanisms, advances in diagnosis and treatment]. Revista de neurologia, 70(12), 453-460. https://doi.org/10.33588/rn.7012.2019474

Serrera-Figallo, M. A., Ruiz-de-León-Hernández, G., Torres-Lagares, D., Castro-Araya, A., Torres-Ferrerosa, O., Hernández-Pacheco, E., \& Gutierrez-Perez, J. L. (2020). Use of Botulinum Toxin in Orofacial Clinical Practice. Toxins, 12(2), 112. https://doi.org/10.3390/toxins12020112.

Schasfoort, F., Dallmeijer, A., Pangalila, R., Catsman, C., Stam, H., Becher, J., Steyerberg, E., Polinder, S., \& Bussmann, J. (2018). Value of botulinum toxin injections preceding a comprehensive rehabilitation period for children with spastic cerebral palsy: A cost-effectiveness study. Journal of rehabilitation medicine, 50(1), 22-29. https://doi.org/10.2340/16501977-2267 